\title{
Comportamento reprodutivo em mulheres ribeirinhas: inquérito de saúde em uma comunidade isolada do Médio Solimões, Amazonas, Brasil
}

\author{
Reproductive behavior in riverside women: health survey in an \\ isolated community in the Middle Solimões, Amazonas, Brazil
}

Ivone Cabra|1, Wilsandrei Cella', Silvia Regina Freitas'

DOI: 10.1590/0103-1104202012709

RESUMO Os inquéritos de saúde conduzidos em comunidades geograficamente isoladas auxiliam na caracterização do comportamento reprodutivo e seus condicionantes. O objetivo deste estudo foi conhecer o perfil da saúde sexual, reprodutiva e aspectos socioculturais e econômicos de mulheres ribeirinhas do Médio Solimões, Amazonas, Brasil. Estudo retrospectivo e descritivo feito com mulheres da comunidade da Vila Sião, Alvarães (AM). No total, 28 mulheres, com idades entre 18 e 57 anos, foram individualmente entrevistadas, utilizando formulário para a obtenção de dados socioculturais, históricos de vida sexual e reprodutiva. Os resultados das análises descritivas indicaram que as entrevistadas eram mestiças, com ensino fundamental, casadas e atuavam na agricultura familiar. Em relação à história de vida sexual das entrevistadas, notou-se que os relacionamentos eram duradouros, com os mesmos parceiros, e as primeiras gestações ocorreram ainda na adolescência. Os indicadores gestacionais mostraram predomínio de parto domiciliar. Essas mulheres apresentaram elevado coeficiente de fecundidade. No entanto, os históricos de perdas gestacionais atribuídos à eclâmpsia, a acidentes domésticos e a doenças enfatizam a importância do acompanhamento pré-natal para a promoção da saúde da gestante e do bebê. A partir desses resultados, conclui-se que a interiorização dos serviços de saúde é essencial para que uma assistência médica especializada chegue até as comunidades ribeirinhas e isoladas do Amazonas, Brasil.

PALAVRAS-CHAVE Inquéritos epidemiológicos. Saúde reprodutiva. População rural.

ABSTRACT Health surveys conducted in geographically isolated communities help to characterize reproductive behavior and its conditioning factors. The aim of this study was to know the sexual and reproductive health profile and the socio-cultural and economic aspects related to riverside women from Middle Solimões, Amazonas, Brazil. This is a retrospective and descriptive study carried out with women from Vila Sião community, Alvarães (AM). Twenty-eight women, 18-57 years old, were individually interviewed using a survey form to obtain socio-cultural data, sexual and reproductive life histories. The results of the descriptive analyses indicated that the respondents were of mixed race, with elementary education, married and worked in family-based agriculture. Regarding the respondents' sexual life history, it was noted that the relationships are long-lasting, with the same partner, and that the first pregnancy occurred during adolescence. Gestational indicators showed a predominance of home birth. These women had a high fertility coefficient. However, the history of gestational loss attributed to eclampsia, home accidents, and illnesses emphasizes the importance of prenatal care for the promotion of the pregnant woman and the baby. From these results it is concluded that the internalization of health services is

1 Universidade do Estado do Amazonas (UEA) - Tefé (AM), Brasil. silvia.sampaio.freitas@ hotmail.com essential for specialized medical assistance to reach the riverside and isolated communities of Amazonas, Brazil.

KEYWORDS Health surveys. Reproductive health. Rural population. 


\section{Introdução}

Desde meados do século XX, o comportamento reprodutivo das mulheres brasileiras tem passado por alterações que induziram seu coeficiente de fecundidade a um declínio significativo ${ }^{1}$. Este fenômeno reflete as mudanças comportamentais e sociais contemporâneas, nas quais o acesso irrestrito a todos os níveis de formação educacional ${ }^{2,3}$, a participação crescente no mercado de trabalho $0^{4}$, a independência financeira ${ }^{5}$, bem como o advento de métodos contraceptivos ${ }^{6}$, facultaram à mulher maior controle sobre suas disposições reprodutivas e opções sobre o número de gestações e o espaçamento entre estas.

No entanto, em contraste com o cenário nacional, as mulheres residentes em regiões isoladas apresentam comportamento reprodutivo compatível com o observado no início do século passado 7 . Isto é, nestas comunidades ainda se verificam precocidade sexual, elevado nível de fecundidade e curto intervalo de tempo entre as gestações ${ }^{8,9}$. No geral, este padrão reprodutivo encontra-se associado a indicadores de baixo desenvolvimento socioeconômico, como atividades de subsistência, baixa escolaridade e acesso limitado a serviços públicos e de saúde ${ }^{10,11}$. No estado do Amazonas, as comunidades isoladas são encontradas em regiões rurais, às margens de lagos e rios, e distantes até $500 \mathrm{~km}$ dos centros urbanos ${ }^{12}$. O isolamento geográfico decorrente da longa distância em relação aos centros urbanos e a proximidade com a floresta favorecem as atividades econômicas de subsistência, como a pesca, o extrativismo e a agricultura familiar ${ }^{13,14}$. Além disto, as comunidades ribeirinhas amazônicas não dispõem de serviços públicos essenciais regulares. Portanto, para acessar os serviços de saúde, centralizados nas sedes municipais, os ribeirinhos sujeitam-se a deslocamentos que podem durar dias ou semanas ${ }^{12,15}$.
As peculiaridades econômicas, sociais e culturais das populações ribeirinhas localizadas no estado do Amazonas contribuem para o insucesso dos programas destinados à saúde da mulher ${ }^{16,17}$. Logo, é imprescindível considerar tais especificidades para elaborar e/ou aperfeiçoar as estratégias de promoção dessas iniciativas ${ }^{\mathbf{1 8}, 19}$. Para tanto, faz-se necessária a realização de inquéritos direcionados a esse público-alvo, a fim subsidiar políticas públicas alinhadas com a cultura e o perfil reprodutivo das mulheres residentes em comunidades amazônicas. Nesse sentido, o presente estudo visou conhecer o perfil da saúde sexual, reprodutiva e aspectos sociais, culturais e econômicos de mulheres ribeirinhas do município de Alvarães, região do Médio Solimões, estado do Amazonas, Brasil.

\section{Material e métodos}

Este estudo retrospectivo e descritivo foi conduzido em julho de 2019, com mulheres adultas e residentes na Vila Sião, Alvarães (AM) (figura 1). Apesar de esta comunidade fazer parte do município de Alvarães (AM), o centro urbano mais próximo fica no município de Tefé (AM), distante $40 \mathrm{~km}$ por via fluvial ${ }^{20}$.

O comércio local é insipiente e dispõe apenas de produtos básicos para alimentação (arroz, macarrão, óleo, açúcar, sal), limpeza (sabão em pó, sabão em barra, cloro, palha de aço) e higiene pessoal (sabonete, pasta e escova de dente, fralda descartável, papel higiênico).

A localidade não possui farmácias, padarias, mercados ou instituições bancárias; os serviços públicos fundamentais (posto de saúde e saneamento básico) também não são oferecidos. A energia elétrica é irregular e provém de uma pequena usina termelétrica. 
Figura1. Vista frontal da Vila Sião, Alvarães (AM).

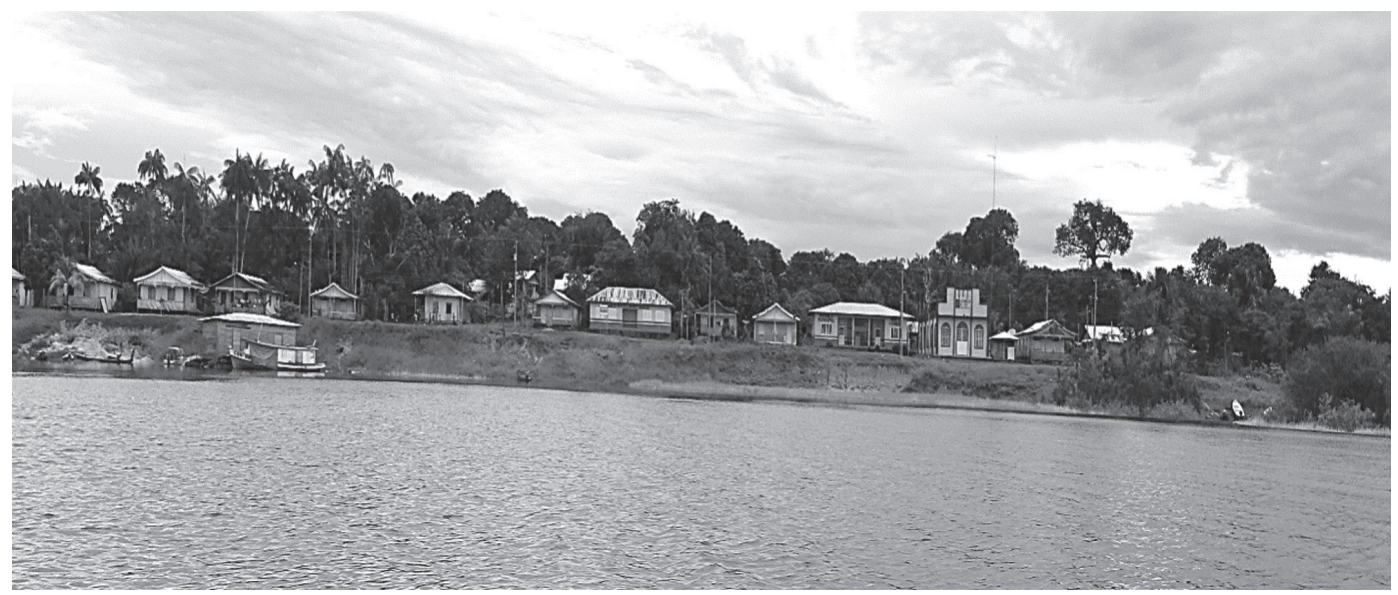

Fonte: Fotografia de Ivone Ketura Silva Cabral, em 10 de agosto de 2019.

A comunidade possui aproximadamente 230 habitantes, organizados em 44 famílias, e é uma das mais populosas das cem comunidades ribeirinhas presentes na Floresta Nacional de Tefé20,21. Os moradores da região têm como meio para sua subsistência a agricultura familiar, com ênfase na produção e comercialização da farinha de mandioca, a pesca artesanal e o extrativismo de andiroba, açaí, castanha e copaíba. Eventualmente, contam com atividades de caça22.

Para a seleção das participantes, foram considerados os seguintes parâmetros: ter 18 anos ou mais, ter iniciado vida sexual e possuir histórico de gestação. Mulheres que não atenderam a um desses parâmetros foram excluídas do estudo.

As entrevistas foram conduzidas por uma única pesquisadora e realizadas nas residências das participantes, onde aplicou-se um formulário semiestruturado para a obtenção de informações sociodemográficas, da vida sexual e da saúde reprodutiva.

As informações sociodemográficas incluíram idade, estado civil (solteira, casada/ união estável, separada/divorciada), etnia autodeclarada (mestiça, negroide, caucasoide), religião, nível de escolaridade (analfabeta, ensino fundamental, ensino médio) e atividade econômica. Para delinear o perfil da vida sexual, avaliou-se o tempo de relacionamento com o parceiro atual ( $<10$ anos; $\geq 10$ anos), o número de parceiros sexuais e o uso de métodos contraceptivos. Na análise da saúde reprodutiva, foram investigadas tanto as condições maternas (idade da menarca, idade da primeira gestação) quanto as condições da gestação (número total de gestações, número de gestações concluídas, número de gestações interrompidas, doenças gestacionais, acompanhamento pré-natal, número de exames de pré-natal, tipo de parto [cesáreo, normal], idade gestacional [pré-termo, a termo]).

As informações obtidas no curso das entrevistas foram organizadas e tabuladas em uma planilha de acesso restrito aos pesquisadores envolvidos neste estudo. Com o auxílio do programa estatístico EpiInfo (versão 3.5.1), foi possível realizar as análises descritivas. As variáveis qualitativas foram expressas em números absolutos e relativos.

Esta pesquisa respeitou os padrões éticos e científicos estabelecidos pela Resolução ${ }^{\circ}$ 466, de 12 de dezembro de 2012, do Conselho Nacional de Saúde/Ministério da Saúde. Seu protocolo foi aprovado pelo Comitê de Ética em Pesquisa da Universidade do Estado do Amazonas, mediante o parecer $\mathrm{n}^{\circ}$ 2.895.249, de 15 de julho de 2018. 
Todas as participantes tomaram ciência dos objetivos do estudo e da confidencialidade das informações prestadas. Após a aceitação do convite para participar do estudo, todas assinaram o Termo de Consentimento Livre e Esclarecido.

\section{Resultado e discussão}

No total, 28 (87,5\%) mulheres adultas, com idades entre 18 e 57 anos, foram entrevistadas. Quatro mulheres foram excluídas do estudo: três não se encontravam na comunidade no período da entrevista e uma, extremamente idosa, não possuía lucidez suficiente para ser entrevistada.

\section{Perfil sociodemográfico e econômico das mulheres ribeirinhas da Vila Sião, Alvarães (AM)}

A análise sociodemográfica (tabela 1) evidencia que as entrevistadas são predominantemente mestiças ( $n=16 ; 57,1 \%)$; estão entre 20 e 33 anos de idade ( $\mathrm{n}=12 ; 42,9 \%)$; têm o ensino fundamental completo ( $\mathrm{n}=22 ; 78,6 \%)$; são casadas ou têm união estável ( $n=26$; 92,6\%); e são evangélicas ( $\mathrm{n}=16 ; 57,1 \%)$. A maioria das entrevistadas $(\mathrm{n}=20 ; 71,4 \%)$ reside na comunidade há mais de 10 anos.

No geral, as mulheres da localidade são jovens e estão em união estável. Os casos de analfabetismo são restritos às mulheres com mais de 40 anos, provavelmente as que não foram beneficiadas pela criação recente de uma unidade escolar de ensino fundamental na comunidade. Nos últimos anos, o investimento em cursos de formação de professores, como o Programa Nacional de Formação de Professores da Educação Básica (Parfor), associado à construção de novas escolas, tem contribuído para a interiorização do ensino básico e a fixação dos professores em comunidades isoladas do estado do Amazonas ${ }^{12}$. Portanto, entende-se que este novo panorama social já esteja refletido no número expressivo de mulheres com ensino fundamental completo.

Tabela 1. Perfil sociodemográfico das mulheres adultas residentes na Vila Sião, Alvarães (AM), 2019

\begin{tabular}{lrr}
\hline Variáveis & Número de Pessoas & Percentual (\%) \\
\hline Etnia & 16 & $57,1 \%$ \\
\hline Mestiça & 3 & $10,7 \%$ \\
Negroide & 9 & $32,1 \%$ \\
Caucasoide & & $25 \%$ \\
\hline Idade Atual & 7 & $42,9 \%$ \\
\hline$<20$ anos & 12 & $32,1 \%$ \\
\hline $20-33$ anos & 9 & $10,7 \%$ \\
$>35$ anos & 3 & $78,6 \%$ \\
\hline Nível de Escolaridade & 22 & $10,7 \%$ \\
\hline Analfabeta & 3 & $3,6 \%$ \\
Ensino Fundamental & & $92,6 \%$ \\
Ensino Médio & 1 & $3,6 \%$ \\
\hline Estado Civil & 26 & 1 \\
\hline Solteira & & \\
Casada & 3 & \\
\hline
\end{tabular}


Tabela 1. (cont.)

\begin{tabular}{lrr}
\hline Variáveis & Número de Pessoas & Percentual (\%) \\
\hline Perfil Religioso & & \\
\hline Católica & 4 & $14,3 \%$ \\
Evangélica & $57,1 \%$ \\
Sem religião & 8 & $28,5 \%$ \\
\hline Tempo de Residência na Comunidade & 7 & $25 \%$ \\
\hline$<10$ anos & 20 & $71,4 \%$ \\
$>10$ anos & 1 & $3,6 \%$ \\
Não lembra & & $35,7 \%$ \\
Perfil Econômico & 10 & $25 \%$ \\
\hline Sem Renda & 7 & $10,7 \%$ \\
<1 salário mínimo & 3 & $28,6 \%$ \\
\hline Não quis responder mínimo & 8 & \\
\hline
\end{tabular}

Fonte: Elaboração própria.

As atividades econômicas predominantes entre as mulheres ribeirinhas da comunidade são a agricultura familiar, baseada na plantação de legumes, verduras e mandioca, e a comercialização da farinha. As atividades de caça e a pesca artesanal estão restritas aos homens da comunidade. A maioria das mulheres entrevistadas declara não ter renda mensal fixa $(\mathrm{n}=10$; $35,7 \%)$. A justificativa informada é que a renda familiar é obtida, exclusivamente, através da venda da farinha de mandioca nos centros urbanos de Tefé (AM) ou Alvarães (AM). Logo, durante os meses de plantio, colheita da mandioca e produção da farinha, a sobrevivência advém da caça, da pesca e daquilo que a terra lhes dá, como frutos e verduras. Entre aquelas que possuem renda mensal, os proventos são decorrentes de pensão pecuniária (1 salário mínimo: $\mathrm{n}=3 ; 10,7 \%$ ) ou de auxílio público social (inferior a 1 salário mínimo: $n=7 ; 25 \%$ ).

A renda familiar insuficiente é amplamente verificada nas comunidades ribeirinhas do estado do Amazonas 12,22,23. Mas, apesar das limitações econômicas, a abundância de alimentos obtidos nos rios locais e na floresta parece atender às necessidades nutricionais das entrevistadas e de seus familiares.

\section{História de vida sexual e as práticas contraceptivas}

Na investigação sobre a história de vida sexual ativa das mulheres da Vila Sião, Alvarães (AM), nota-se que os relacionamentos são duradouros - acima de 10 anos ( $n=15 ; 54 \%)$ - e com os mesmos parceiros ( $\mathrm{n}=21 ; 75 \%$ ) (tabela 2). Esse comportamento destoa do observado tanto em povos indígenas ${ }^{24}$ quanto em populações urbanas $^{25}$. Neste sentido, a união conjugal precoce, duradoura e sem trocas de parceiros se apresenta como um comportamento cultural e prevalente em algumas comunidades ribeirinhas e isoladas do Amazonas ${ }^{6}$.

Ainda que as mulheres entrevistadas estejam em relacionamentos socialmente estáveis, a maioria declara não ter intenção de engravidar ( $n=20 ; 71,4 \%)$. Esta opção é apresentada pelas entrevistadas independentemente do número de filhos que possuem. Por este motivo, 92,8\% ( $\mathrm{n}=26)$ adotam algum tipo de estratégia contraceptiva. Os contraceptivos hormonais orais $(n=4 ; 14,3 \%)$, os injetáveis $(\mathrm{n}=4 ; 14,3 \%)$ e o preservativo masculino $(\mathrm{n}=3$; $10,7 \%)$ são citados pela maioria das mulheres. No entanto, a adesão a tais práticas está 
condicionada à disponibilidade destes dispositivos para a comunidade da Vila Sião, Alvarães (AM). O único posto de saúde da comunidade encontra-se inoperante. Como consequência, as mulheres da localidade só têm acesso aos contraceptivos comerciais quando recebem a visita de agentes do Programa Saúde da Família, ou, mais raramente, quando viajam para os centros urbanos de Tefé (AM) ou Alvarães (AM). Os anticoncepcionais orais/ injetáveis e os métodos de barreira (preservativo masculino/feminino) são essenciais para o controle da natalidade, assim como para a prevenção de infecções transmitidas por via sexual ${ }^{26}$. Contudo, a irregularidade no fornecimento destes dispositivos, por limitações logísticas, compromete o controle eficiente da concepção e de possíveis infecções sexuais nas mulheres da Vila Sião, Alvarães (AM).

Como alternativa aos métodos convencionais de contracepção, 28,6\% (n=8) das entrevistadas fazem uso regular de chás caseiros. Os chás à base de ervas nativas é uma prática reconhecida entre as mulheres de comunidades amazônicas isoladas ${ }^{1,27} \mathrm{e}$ indígenas ${ }^{24}$. Entre as ervas nativas indicadas pelas entrevistadas, destacam-se: saracura-mirá, carapanaúba, sara-tudo, boldo, algodão e copaíba. Estudos bioquímicos e farmacológicos conduzidos nos extratos das plantas citadas pelas entrevistadas mostram a presença de moléculas com ação contraceptiva ${ }^{28-30}$. Conhecer as práticas tradicionais de controle da concepção, como o uso de chás à base de ervas naturais, é um diferencial dos profissionais da saúde que atuam em comunidades isoladas. Esta qualificação do profissional viabiliza a integração dos saberes culturais com as práticas médicas de cuidado com o corpo, configurando uma estratégia factível para a promoção da saúde sexual das mulheres ribeirinhas.

Entre aquelas que não fazem uso de métodos contraceptivos, $25 \%(\mathrm{n}=7)$ informaram ter realizado a laqueadura tubária. A opção por esse método de contracepção deu-se por vontade das próprias mulheres, com o intuito de terem famílias menores. Elas alegam que a esterilização é a medida mais eficiente para o controle reprodutivo, considerando a irregularidade do acesso aos métodos contraceptivos tradicionais (preservativo masculino, contraceptivos orais/injetáveis).

Neste estudo, o percentual de mulheres que não utilizam qualquer tipo de método contraceptivo é de 7,1\% (n=2). O tamanho da prole reflete um comportamento social relatado em outras populações isoladas da região do Médio Solimões, Amazonas, Brasi16,24,31. Nestes casos, o acompanhamento pré-natal torna-se essencial para orientar sobre o período mínimo intergestacional. De acordo com as Diretrizes da Sociedade Brasileira de Ginecologia e Obstetrícia, a mulher deve considerar um período de 18 meses entre as gestações, a fim de assegurar um desenvolvimento adequado às gestações futuras ${ }^{32}$.

Com base nos parâmetros investigados, sugere-se que as mulheres da Vila Sião, Alvarães (AM), estejam em um processo de transição sociocultural, na qual a estrutura familiar com poucos filhos seja privilegiada. No entanto, para a confirmação desta hipótese, faz-se necessária a realização de estudos de follow-up, bem como o acompanhamento das futuras gerações.

Tabela 2. Perfil da vida sexual das mulheres adultas residentes na Vila Sião, Alvarães (AM), 2019

\begin{tabular}{lrr}
\hline Variáveis & Número de Pessoas & Percentual (\%) \\
\hline Tempo de Relacionamento & & \\
\hline$<10$ anos & 13 & $46 \%$ \\
$\geq 10$ anos & 15 & $54 \%$ \\
\hline
\end{tabular}




\begin{tabular}{lrr}
\hline Tabela 2. (cont.) & Número de Pessoas & Percentual (\%) \\
\hline Variáveis & & \\
\hline Número de Parceiros & 21 & $75 \%$ \\
\hline Apenas 1 parceiro & 7 & $25 \%$ \\
$>1$ parceiro & & $28,6 \%$ \\
\hline Intenção de Engravidar & 8 & $71,4 \%$ \\
\hline Sim & 20 & $39,3 \%$ \\
Não & & $28,6 \%$ \\
\hline Uso de Métodos Contraceptivos & 11 & $25 \%$ \\
\hline Métodos Convencionais & 8 & $7,1 \%$ \\
Métodos Caseiros & 7 & 2 \\
Esterilização Feminina & & \\
Não utiliza contraceptivos & & \\
\hline
\end{tabular}

Fonte: Elaboração própria.

\section{Saúde reprodutiva e seus indicadores maternos e da gestação}

As entrevistadas atingiram a menarca entre 11 e 13 anos ( $n=26 ; 92,9 \%)$, conforme apresentado na tabela 3. Este parâmetro encontra-se em conformidade com o Consenso da Federação Brasileira das Associações de Ginecologia e Obstetrícia ${ }^{32}$. De acordo com este documento, a primeira menstruação ocorre entre 10 e 16 anos, com idade média de 12,3 anos. Portanto, a menarca está condicionada à melhoria nas condições de vida e saúde da mulher ${ }^{32}$.

A idade da primeira gestação ocorreu com maior frequência entre 14 e 15 anos ( $\mathrm{n}=19$; 67,9\%). Somando-se os grupos etários 14-15 anos e 16-17 anos, observa-se que $78,6 \%$ das mulheres iniciaram o ciclo reprodutivo ainda na adolescência. Esta tendência de concentração da fecundidade nas idades mais jovens do período reprodutivo é frequentemente observada em grupos vulneráveis, cujas idades médias na primeira gestação variam de 16 a 1733. É notório que a gravidez precoce pode gerar riscos, tanto para a saúde da mãe quanto para o bebê, como consequência da imaturidade funcional do corpo da adolescente ${ }^{34}$. A fim de minimizar os efeitos adversos da gravidez precoce, estudos apontam para a adoção de medidas de educação reprodutiva. Isto é, ações que visam educar e esclarecer sobre a puberdade, alterações hormonais, ciclo menstrual, métodos contraceptivos, fecundação e gestação ${ }^{34,35}$. De fato, a literatura especializada já apresenta resultados exitosos de programas de educação reprodutiva conduzidos tanto em escolas ${ }^{36,37}$ quanto em unidades básicas de saúde ${ }^{34}$. Frente aos vários relatos de sucesso, torna-se relevante a inclusão da educação reprodutiva como tema transversal nas escolas da rede básica de ensino, como também a criação de programas de saúde com ênfase neste tema. No entanto, a formatação da educação reprodutiva deve considerar as especificidades culturais e locais do público-alvo; neste caso, as comunidades ribeirinhas e isoladas da região do Médio Solimões, Amazonas, Brasil. 
Tabela 3. Indicadores maternos incluídos na análise do perfil reprodutivo das mulheres residentes na Vila Sião, Alvarães (AM), 2019

\begin{tabular}{lrr}
\hline Indicadores Maternos & Número de Pessoas & Percentual \% \\
\hline Idade da Menarca & 26 & \\
\hline Entre 11 e 13 anos & 2 & $92,9 \%$ \\
Entre 14 e 15 anos & & $7,1 \%$ \\
\hline Idade na Primeira Gestação & 19 & $67,9 \%$ \\
\hline 14 a 15 anos & 3 & $10,7 \%$ \\
16 a 17 anos & 6 & $21,4 \%$ \\
\hline
\end{tabular}

Fonte: Elaboração própria.

Ao avaliar os indicadores gestacionais (tabela 4), verificou-se o predomínio do parto vaginal, ocorrido na residência da parturiente e com o auxílio de parteiras $(\mathrm{n}=17 ; 61 \%)$. $\mathrm{Na}$ comunidade citada, apenas as gestantes com risco que justifique o parto cesáreo são encaminhadas para os serviços hospitalares disponíveis nos centros urbanos de Tefé (AM) ou Alvarães (AM), recorrendo ao parto hospitalar.

No Brasil, a modalidade de parto natural, domiciliar e acompanhado por parteiras experientes ainda é associada à falta de recursos econômicos e à dificuldade de acesso aos serviços de saúde ${ }^{38}$. No entanto, iniciativas recentes do Ministério da Saúde vêm norteando ações de humanização do parto. Neste sentido, conquistas como a criação dos Centros de Parto Normal (1999) ${ }^{39}$, do Programa Nacional de Humanização do Pré-Natal e Parto $(2000)^{40} \mathrm{e}$ da Rede Cegonha (2011) ${ }^{41}$ consolidam o modelo de assistência obstétrica para a humanização do parto no País ${ }^{38}$. A literatura recente sobre o assunto aponta que mulheres das regiões Sul e Sudeste, com elevado nível de escolaridade, estão buscando serviços privados para o parto domiciliar planejado. Neste contexto, doulas e enfermeiras obstétricas configuram profissionais habilitadas para desempenhar o acompanhamento domiciliar ${ }^{\mathbf{3 8}}$.

No momento atual da saúde da mulher, em que se preconiza o parto humanizado e domiciliar, aliado à fragilidade dos serviços públicos de saúde em comunidades isoladas, é fundamental que os gestores em saúde dos municípios amazônicos consolidem ações que visem à capacitação de novas parteiras, assim como à atualização e orientação das parteiras profissionais.

No tocante à idade gestacional, $86 \%(n=24)$ dos nascimentos ocorreram entre a $37^{\mathrm{a}}$ e a $41^{\mathrm{a}}$ semana de gestação. Este índice encontra-se abaixo do calculado para a região urbana do município de Tefé (AM), que foi de 93,07\%, para o período de 2006 a $2012^{31}$. Neste contexto, o índice elevado de nascimentos pré-termo é preocupante e aponta a urgência de aperfeiçoamento dos protocolos do acompanhamento de pré-natal, considerando as especificidades sociais e culturais das comunidades rurais isoladas, em especial da Vila Sião, Alvarães (AM).

Essas mulheres apresentaram elevado coeficiente de fecundidade, que variou de duas $(\mathrm{n}=8 ; 28,6 \%)$ a mais de cinco gestações concluídas $(n=10 ; 35,7 \%)$. No parâmetro perda gestacional, 53,6\% ( $n=15)$ afirmaram já ter sofrido dois ou mais abortos. As interrupções das gestações foram atribuídas à eclâmpsia, a acidentes domésticos e doenças em geral. Estes dados enfatizam a importância do acompanhamento pré-natal para a promoção da saúde da gestante e do bebê. De fato, a realização de consultas e exames complementares durante $o$ desenvolvimento da gravidez é essencial para a proteção e prevenção de eventos adversos, 
permitindo a identificação e o manuseio clínico de intervenções adequadas sobre potenciais fatores obstétricos de risco ${ }^{\mathbf{4 2}, 43}$.

Os indicadores gestacionais investigados neste estudo sinalizam que as mulheres da comunidade da Vila Sião, Alvarães (AM), possuem fatores de risco que não podem ser desconsiderados. A ocorrência de nascimentos prematuros, os relatos de perda gestacional e o reconhecimento de doenças maternas (eclâmpsia) são preditores de risco passíveis de controle. No entanto, a preocupação com o bem-estar das gestantes/parturientes e sua prole precisa ser consolidada. A interiorização dos serviços de saúde é essencial para que o cuidado e a assistência médica especializada cheguem às comunidades ribeirinhas e isoladas. Afinal, uma parte significativa da população do estado do Amazonas reside em comunidades, povoados, tribos em situação de isolamento geográfico.

Tabela 4. Indicadores gestacionais incluídos na análise do perfil reprodutivo de mulheres residentes na Vila Sião, Alvarães (AM), 2019

\begin{tabular}{lrr}
\hline Indicadores Gestacionais & Número de Pessoas & Percentual (\%) \\
\hline Tipo de Parto & & \\
\hline Vaginal & 17 & $61 \%$ \\
Cesariana & 2 & $7 \%$ \\
Ambos & 9 & $32 \%$ \\
\hline Idade Gestacional & 24 & $86 \%$ \\
\hline A termo & 4 & $14 \%$ \\
\hline Pré-termo & & $28,6 \%$ \\
\hline Paridade & 8 & $35,7 \%$ \\
\hline 2 filhos & 10 & $35,7 \%$ \\
\hline e 4 filhos & 10 & \\
\hline 5 filhos & & $53,6 \%$ \\
\hline Histórico de Perda Gestacional & 15 & $46,4 \%$ \\
\hline Não & 13 & \\
\hline
\end{tabular}

Fonte: Elaboração própria.

Como limitação da pesquisa, aponta-se a escassez de estudos científicos qualificados, voltados para os grupos populacionais negligenciados, como as populações ribeirinhas do estado do Amazonas. O ambiente atípico, com densa floresta tropical, imensos rios e chuvas torrenciais, restringe a aquisição de informações de caráter científico por pesquisadores. A carência de informação frustra a realização de análises comparativas mais abrangentes quanto aos indicadores de saúde e reprodutivos de mulheres em situação de isolamento geográfico. Neste aspecto, o desenvolvimento de estudos no ambiente amazônico exige suporte de recurso financeiro e esforço adicional em relação aos desenvolvidos em áreas urbanas. Desta maneira, espera-se que os resultados do presente estudo possam contribuir para o desenvolvimento de futuras investigações entre os povos ribeirinhos localizados no estado do Amazonas e balizar aspectos que permitam a articulação de políticas públicas para esses povos. 


\section{Conclusões}

Os resultados do presente estudo apontam que o comportamento reprodutivo das mulheres da Vila Sião, Alvarães (AM), ocorre sob condições de risco. Os indicadores sociais e gestacionais evidenciam um padrão de reprodução caracterizado por gestação precoce, elevada taxa de fecundidade e perdas gestacionais. Tal perfil sinaliza que as mulheres ribeirinhas se reproduzem com gravidezes de risco para si e para seus filhos, uma vez que, na maioria dos casos, não atendem ao período mínimo intergestacional de 18 meses, estabelecido pelos parâmetros da medicina moderna. Quase todos os partos são realizados sem assistência pré-natal, e, em poucos casos, a mulher planeja a sua reprodução.

Ressaltam, ainda, que a atenção primária fundamentada no modelo medicalizante e hospitalocêntrico distancia as populações ribeirinhas, e geograficamente isoladas, do atendimento à sua saúde. No entanto, a criação de programas para a atenção humanizada do pré-natal e de planejamento familiar, assim como investimentos na formação, capacitação e atualização de profissionais de saúde especializados, poderão assegurar um atendimento de qualidade às mulheres ribeirinhas. Aliás, os programas de acompanhamento e planejamento familiar devem nortear a atenção humanizada, considerando os valores culturais e saberes populares das comunidades ribeirinhas e isoladas.

Por fim, ressalta-se a necessidade de aprimoramento e expansão dos serviços de atendimento pré-natal, de sensibilização sobre os riscos da eclâmpsia para a saúde materna, bem como da criação de programas de saúde reprodutiva que reconheçam as condições sociais e culturais das comunidades isoladas existentes no estado do Amazonas.

\section{Colaboradores}

Cabral I ( 000-0002-0328-4745)* contribuiu substancialmente para a concepção, o planejamento, a análise e a interpretação dos dados; contribuiu significativamente para a elaboração do rascunho e da revisão crítica do conteúdo; e participou da aprovação da versão final do manuscrito. Cella W (00000002-8107-3062)* contribuiu substancialmente para a concepção e o planejamento do estudo; contribuiu significativamente para a revisão crítica do conteúdo; e participou da aprovação da versão final do manuscrito. Freitas SR (0000-0003-2987-7837)* contribuiu substancialmente para a análise e a interpretação dos dados; contribuiu significativamente para a elaboração do rascunho e da revisão crítica do conteúdo; e participou da aprovação da versão final do manuscrito. 


\section{Referências}

1. Wong LLR, Morell MGG, Carvalho RL. Notas sobre o comportamento reprodutivo da população autodeclarada indígena - Censos Demográficos 1991 e 2000. Rev. Bras. Estudos Pop. 2009; 26(1):61-75.

2. Horta CJ, Carvalho JAM, Nogueira OJO. Evolução do Comportamento Reprodutivo da Mulher Brasileira 1991-2000. Cálculo da Taxa de Fecundidade Total em Nível Municipal. Rev. Bras. Estudos Pop. 2005; 22(1):131-140.

3. Fundação Sistema Estadual de Análise de Dados Natalidade e Fecundidade em São Paulo. O Risco da Interpretação Equivocada dos Dados. São Paulo: SP Demográfico; 2004.

4. Dias CS. Diferenciais no Comportamento Reprodutivo das Mulheres Brasileiras: Uma Análise a partir dos Grupos Ocupacionais. Rev. Ciênc. Soc. 2008; 53(1):233-265.

5. Alves JED. Questões Demográficas: Fecundidade e Gênero. Rio de Janeiro: Escola Nacional de Ciências Estatísticas; 2004.

6. Moura EAF. Comportamento reprodutivo das mulheres ribeirinhas do Amanã. Uakari. 2005; 1(1):31-39.

7. Gupta N, Leite IC. Tendências e determinantes entre adolescentes do Nordeste do Brasil. Persp. Inter. Plan. Fam. 2001; 45(esp):24-29.

8. Villela TF, Vieira EM, Gonçalves TC, et al. Declaração de nascidos vivos no município de Volta Redonda, RJ: perfil da gestante adolescente. Cad. UniFOA. 2018; 6(2):59.

9. Rodrigues ARS, Barros WM, Soares PDFL. Reincidência da gravidez na adolescência: percepções das adolescentes. Enf. Foco. 2016; 7(3/4):66-70.

10. Ruzany MH, Moura EAF, Meirelles ZV. Adolescentes e jovens de populações ribeirinhas na Amazônia - Brasil. Rio de Janeiro: Visão Social; 2012.
11. Oliveira RS. Os "filhos da mãe" no Médio Solimões. In: $13^{\circ}$ Anais do Seminário Internacional Fazendo Gênero; 2013 set 16-20; Florianópolis. Florianópolis: UFSC; 2012.

12. Gama ASM, Fernandes TG, Parente RCP, et al. Inquérito de saúde em comunidades ribeirinhas do Amazonas, Brasil. Cad. Saúde Pública. 2018; 34(2):17-22.

13. Lima DM. Ribeirinhos, Pescadores e a Construção da Sustentabilidade nas Várzeas dos Rios Amazonas e Solimões. Boletim Rede Amazônia. 2004; 3(1):57-66.

14. Loureiro JJ. A Amazônia no século XXI: novas formas de desenvolvimento. São Paulo: Editora Empório do Livro; 2009.

15. Cabral IKS, Cella W, Freitas SRS. Reproductive behavior of women from a rural community in Tefé, Amazonas, Brazil. Braz. J. Biol. Sciences. 2019; 6(14):497504.

16. Bôas LMS, Oliveira DC. A saúde das comunidades ribeirinhas da região Norte brasileira: Revisão sistemática da literatura. Invest. Qualitat. Saúde. 2016; 2(3):62-71.

17. Franco EC, Santo CE, Arakwa AM, et al. Promoção da população ribeirinha da região Amazônica: Relato de experiência. Rev. CEFAC. 2015; 17(5):1521-1530.

18. Costa MCO. Indicadores materno-infantis na adolescência e juventude: sociodemográfico, pré-natal, parto e nascidos-vivos. J. Pediat. 2001; 77(3):235-242.

19. Mascarenhas MDM, Rodrigues MTP, Monte NF. Caracterização dos partos e dos nascidos vivos em Piripiri, Piauí, Brasil. Rev. Bras. Saúde Mat. Infant. 2006; 6(2):175-181.

20. Instituto Chico Mendes de Conservação da Biodiversidade. Plano de manejo da Floresta Nacional de Tefé: diagnósticos. Brasília, DF: ICMBIO; 2014.

21. Brianezi A. Floresta Nacional de Tefé: encontros e 
desencontros das políticas ambiental e agrária na Amazônia. Rev. IDEAS. 2008; 2(2):256-283.

22. Oliveira MG, Suertegaray DMA, Pires CLZ. Mapeamento participativo e uso do SIG: FLONA de Tefé-AM. In: Anais do $16^{\circ}$ Simpósio Brasileiro de Sensoriamento Remoto; 2013 abr 18; Foz do Iguaçu. Foz do Iguaçu: INPE; 2013.

23. Piperata BA, Spence JE, Dagloria P, et al. The nutrition transition in Amazonia: rapid economic change and its impact on growth and development in ribeirinhos. Am. J. Physiol. Anthr. 2011; 1(46):1-13.

24. Anunciação-Lima CM, Alcântara KD, Cerqueira JML, et al. Iniciação sexual, gestação, parto e puerpério em comunidades indígenas do Brasil: uma breve revisão integrativa. Rev. Saúde Púb. 2018; 1(1):86-101.

25. Abramovay M, Castro MG, Silva LB. Juventude e sexualidade. Brasília, DF: UNESCO; 2004.

26. Ricci AP, Sene AG, Souza BLB, et al. Infecções sexualmente transmissíveis na gestação: educação em saúde como estratégia de prevenção na atenção básica/ Sexually transmitted infections during pregnancy: health education as a prevention strategy in primary care. Braz. J. Health Rev. 2019; 2(1):565-570.

27. Silva LR, Silva RF. Conhecimento, atitudes e crenças de mulheres ribeirinhas frente à concepção e contracepção. Rev. Enfer. 2009; 3(4):72-80.

28. Ribeiro JELS. Guia de identificação das plantas vasculares de uma floresta de terra firme na AM central. 19. ed. Manaus: Midas Printing; 1999.

29. Silva RJB, Oliveira APS, Silva RLF. Crenças populares: Atribuições místicas e medicinais às plantas na baixada cuiabana, Mato Grosso, Brasil. Biodivers. 2018; 17(1):61.

30. Vásquez SPF, Mendonça MS, Noda SN. Etnobotânica de plantas medicinais em comunidades ribeirinhas do Município de Manacapuru, Amazonas, Brasil. Acta Amaz. 2014; 44(4):457-472.
31. Cella W, Marinho KO. Delineamento epidemiológico dos nascidos vivos em Tefé, Amazonas, no período de 2006 a 2012. Arq. Ciênc. Saúde UNIPAR. 2017; 21(2):99-104.

32. Cabral ZAF. Manual de Genecologia infanto juvenil. São Paulo: Federação brasileira de ginecologia e obstetrícia; 2014.

33. Ruzany MH, Andrade CLT, Meireles ZV, et al. Desinformação e vulnerabilidades com relação à sexualidade dos adolescentes e jovens da Reserva de Mamirauá, Amazonas - Brasil. Adolesc. Saúde. 2010; 7(2):41-49.

34. Oliveira MJP, Lanza LB. Educação em saúde: doenças sexualmente transmissíveis e gravidez na adolescência. Rev. Facul. Ciências Méd Sorocaba. 2018; 20(3):138-141.

35. Franze AMAK, Benedet DCF, Wall ML, et al. Planejamento reprodutivo nas orientações em saúde: revisão integrativa. REFACS. 2019; 7(3):366-377.

36. Malta DC, Machado ÍE, Felisbino-Mendes MS, et al. Uso de substâncias psicoativas em adolescentes brasileiros e fatores associados: Pesquisa Nacional de Saúde dos Escolares, 2015. Rev. Bras. Epidemiol. 2018; (21):e180004.

37. Duarte ES, Pamplona TQ, Rodrigues AL. A gravidez na adolescência e suas consequências biopsicossociais. DeCiência FOCO. 2018; 2(1):45-52.

38. Castro CM. Os sentidos do parto domiciliar planejado para mulheres do município de São Paulo, São Paulo. Cad. Saúde Col. 2015; 23(1):69-75.

39. Campos SEV, Lana FCF. Resultados da assistência ao parto no Centro de Parto Normal Dr. David Capistrano da Costa Filho em Belo Horizonte, Minas Gerais, Brasil. Cad. Saúde Pública. 2007; 23(6):1349-1359.

40. Gligio MRP, França E, Lamouner JA. Avaliação da qualidade da assistência ao parto normal. Rev. Bras. Ginecol. Obst. 2011; 33(10):297-304. 
41. Brasil. Ministério da Saúde. Departamento de Ações Programáticas Estratégicas. Gestação de alto risco: manual técnico. Brasília, DF: Ministério da Saúde; 2012.

42. Basso CG, Neves ET, Silveira A. Associação entre realização de pré-natal e morbidade neonatal. Contex. Enf. 2012; 21(2):76-269.
43. Brasil. Ministério da Saúde. Pré-natal e puerpério: atenção qualificada e humanizada: manual técnico. Brasília, DF: Ministério da Saúde; 2006.

Recebido em 09/12/2019

Aprovado em 18/09/2020

Conflito de interesses: inexistente

Suporte financeiro: Fundação de Amparo à Pesquisa do Estado do Amazonas (Fapeam). Edital Paic/Fapeam/UEA no 021/2018 e Sisproj no 14404 